\title{
New model predicts the risk of infective endocarditis in adulthood
}

A new model based on six clinical parameters can be used to predict the risk of developing infective endocarditis (IE) in later life in young adults with congenital heart disease (CHD).

The population of adults with $\mathrm{CHD}$ is growing and a large proportion develop disease-related complications, including IE, which substantially increases morbidity and mortality. Nevertheless, methods to evaluate risk of developing IE in adulthood are lacking, meaning that long-term medical plans cannot be made for young adults.

Barbara Mulder and colleagues, who developed the model, used a registry of over 12,000 patients with CHD in the Netherlands that was set up to enable the evaluation of long-term outcomes in adults. The researchers measured the occurrence of IE up to 40 years of age.

Patient sex, the type of main congenital heart defect, multiple heart defects, and three types of complications in childhood were shown to predict risk of IE and were included in the model. Less than $1 \%$ of patients aged $<40$ years who were classified as having low risk ( $<3 \%$ risk as predicted by the model) developed IE, whereas the incidence of this complication was $6 \%$ in 'high-risk' patients ( $\geq 3 \%$ risk).

"Our findings may lead to individually tailored medical surveillance and educational counseling," says Mulder, "thus averting IE or enabling timely detection in adult patients with CHD."

The researchers now plan to develop a risk score to predict reoperations in adults with $\mathrm{CHD}$, and are also analyzing predictors of health-care use and risk of sudden cardiac death in this population.

Ana Mateus

Original article Verheugt, C. L. et al. Turning 18 with congenital heart disease: prediction of infective endocarditis based on a large population. Eur. Heart J. doi:10.1093/eurheart/ehq485 